\title{
Inteligência social
}

\author{
Fabiano de Abreu Agrela Rodrigues ${ }^{1}$ \\ contact@CPAHscientificjournal.com
}

\section{RESUMO}

Interagir com as pessoas de maneira positiva não é uma tarefa fácil. Há todo o momento e por diferentes razões, conflitos surgem e provocam rupturas entre as pessoas, muitas delas difíceis de solucionar. Para melhor interação entre os indivíduos é fundamental o desenvolvimento da inteligência social que resulta na interação inteligente, na autoconfiança e no desenvolvimento de habilidades sociais importantes. O presente artigo tem como objetivo apresentar o conceito de inteligência social, suas características e os resultados positivos da sua aplicação.

Palavras-chaves: inteligência social; neurociência; competência; cognição.

\footnotetext{
${ }^{1} \mathrm{PhD}$, neurocientista, mestre psicanalista, biólogo, historiador, antropólogo, com formações também em neuropsicologia, psicologia, neurolinguística, neuroplasticidade, inteligência artificial, neurociência aplicada à aprendizagem, filosofia, jornalismo e formação profissional em nutrição clínica - Diretor do Centro de Pesquisas e Análises Heráclito; Chefe do Departamento de Ciências da Logos University; Membro da Federação Europeia de Neurociências e da Sociedade Brasileira e Portuguesa de Neurociências. Universidades em destaque: Logos University, Nova de Lisboa, Faveni, edX Harvard, Universidad de Madrid. E-mail: contact@CPAHscientificjournal.com
} 


\title{
Social intelligence
}

\begin{abstract}
Interacting with people in a positive way is not an easy task. Conflicts arise all the time and for different reasons, and cause disruptions between people, many of which are difficult to resolve. For a better interaction between individuals it is fundamental to develop social intelligence that results in intelligent interaction, self-confidence, and the development of important social skills. This article aims to present the concept of social intelligence, its characteristics, and the positive results of its application.
\end{abstract}

Keywords: social intelligence ; neuroscience ; competence ; cognition.

Artículo recibido: 05 octubre. 2021 Aceptado para publicación: 02 noviembre 2021 Correspondencia: contact@CPAHscientificjournal.com Conflictos de Interés: Ninguna que declarar 


\section{INTRODUÇÃO}

O conceito da inteligência e sua formação e desenvolvimento foram estudados pelo psicólogo inglês Charles Spearman que cunhou o termo Inteligência Geral para designar habilidades gerais, relacionadas a áreas específicas do cérebro, e ao desenvolvê-las é possível aprimorar habilidades específicas (Rodrigues, 2021).

Tais habilidades gerais estão alicerçadas em duas inteligências especificas. Uma é a inteligência fluída, ela está associada a memória, raciocínio, adaptação, resolução de problemas e atenção e se correlaciona com a área da superfície cortical no parietal superior esquerdo e direito, e temporal inferior esquerdo, inferior direito e temporal médio.

Já a inteligência cristalizada está relacionada com a competência comunicativa e a utilização de experiência passada para resolver problemas e ela está correlacionada com a espessura cortical no centro frontal esquerdo caudal, para-opercular esquerdo, centro frontal esquerdo rostral, parietal superior esquerdo (Tadayon, 2020)

Portanto, a inteligência social é a capacidade do indivíduo de utilizar as funções cognitivas relacionadas a inteligência no mundo externo e se relacionar com os outros e com os ambientes de maneira inteligente desenvolvendo a inteligência interpessoal, o controle emocional, a comunicação verbal e o reconhecimento da comunicação não verbal, habilidade de observação mais apurada (Kihlstrom e Cantor, 2000).

\section{INTELIGÊNCIA SOCIAL}

A inteligência é vista por muitos estudiosos como um aspecto evolucionário na espécie humana, desde nossas origens o homem vêm recebendo estímulos do ambiente que provocam mudanças físicas e comportamentais que são passadas de uma geração para outras tanto pela via cultural quanto pela hereditariedade através das informações do nosso DNA.

Ela pode ser definida de maneira geral a capacidade que o indivíduo possui de resolver problemas se utilizando tanto de experiências passadas e encontrando padrões similares entre elas, quanto por adaptação, encontrado vias diferentes para resolver algo de maneira diferente de como era anteriormente resolvida.

A origem dessas capacidades como a memória, o raciocínio lógico, a capacidade para comunicação verbal e o reconhecimento de símbolos que culminou no aprendizado da leitura, está localizada no córtex cerebral que durante a evolução humana passou por 
processos de mudanças significativas, como o aumento do cérebro, as mudanças e o desenvolvimento de suas estruturas internas, o aumento das sinapses que mandam informação para as outras áreas do cérebro e o aumento da quantidade de neurônios (Weeb et al, 2014).

Segundo pesquisas, o início das interações sociais se deu no período de desenvolvimento do Homo Erectus. Essa espécie hominídea apresentava características que a diferenciava dos outros primatas como o bipedalismo, a capacidade de utilizar ferramentas e o uso do fogo para proteção, aquecimento e alimentação. Esse período é caracterizado pela união da espécie em pequenas comunidades cooperativas demonstrando comportamentos coletivos similares ao do homem moderno.

Entre esses comportamentos estava a caça conjunta e a imigração para áreas com melhor clima, maiores opções de alimentação e o início da cooperação masculina. Todas essas mudanças são resultado da busca pela sobrevivência e a percepção que o grupo sobrevive melhor do que o indivíduo solitário (Hatala et al 2016).

Essa capacidade de se relacionar e interagir de forma inteligente são resultados do desenvolvimento de uma série de traços comportamentais aprendidos durante a história da evolução humana e continua a se desenvolver durante a vida. De acordo com Boyatzis (2017) pessoas que possuem essa inteligência apresentam comportamentos distintos das outras pessoas e são extremamente requisitadas no mercado de trabalho. Alguns desses comportamentos são:

- Autocontrole: É responsável pela construção de uma vida equilibrada e de bem estar. Seu desenvolvimento influencia as funções cognitivas, impossibilitando o aparecimento de um comportamento impulsivo e desenfreado. Ele melhora as relações interpessoais e ajuda nas conquistas acadêmicas e profissionais. Pessoas que desenvolvem autocontrole são menos propensas a comportamentos compulsivos como gastos exacerbados e má alimentação, além de serem menos procrastinadoras (Gillebaart, 2018).

- Adaptação: É a capacidade de observar o ambiente, reter informações e identificar sinais de conflito, perturbação, discordância ou perigo ali apresentados ajustando comportamentos e ações para melhorar as relações interpessoais e resolver problemas usando novas estratégias. Essa habilidade é utilizada desde o princípio da evolução 
humana para a sobrevivência da espécie e permitiu as mudanças físicas, cognitivas e sociais dos humanos

- Empatia: É a capacidade de reconhecer e entender, através da comunicação verbal e não verbal, os sentimentos dos outros, seus desejos, suas motivações, medos, inseguranças e como o outro vê o mundo. Ela permite liderar os outros, administrar situações diversas e lidar com conflitos (Badea, 2010).

- Inspiração: É a capacidade de exercer uma influencia positiva nas outras pessoas e conduzi-las em direção a um objetivo em comum aperfeiçoando suas capacidades, trazendo uma renovação da auto-estima e oferecendo a elas uma ambiente saudável onde se sentem valorizadas e reconhecidas (Chopra e Kanji, 2010).

- Trabalho em equipe: Habilidade de trabalhar com pessoas com diferentes personalidades, conhecimentos e temperamentos para alcançar um objetivo em comum. Os fatores mais importantes para um bom trabalho em equipe são coordenação, assertividade, liderança, distribuição de tarefas em acordo com o conhecimento e habilidades de cada membro do grupo, apoio mútuo e troca de informações (Westli et al, 2010).

- Administrar conflitos: O conflito é descrito como o desentendimento e incompatibilidade de ideias entre uma ou mais partes em relação a alguma decisão importante e está presente em todas as esferas das relações humanas. Aprender a resolver conflitos é uma habilidade importante que produz um ambiente saudável e com resultados justos e eficazes (Abas et al, 2010).

- Orientação para resultados: É a capacidade de se manter firme em alcançar um objetivo, sem se desviar por caminhos que não apresentam os melhores resultados, manter a si mesmo e os outros focados, melhorar o próprio desempenho, planejar, desenvolver estratégias eficazes, se organizar e concretizar atividades com empenho e dedicação (Alhadabi e Karpinski, 2020).

A palavra-chave é competência. Ela é a capacidade de tomar decisões e de ter habilidades variadas que conduzam o próprio indivíduo e os outros para um alto desempenho. Essa é uma habilidade importante, principalmente nos ambientes de trabalho, pois indicam a capacidade para a flexibilidade, aprendizagem e intencionalidade.

Ter em mente a intencionalidade das próprias ações permite um melhor conhecimento de si mesmo e uma melhor orientação dos próprios comportamentos. Por exemplo, escutar 
e fazer perguntas para uma pessoa com a intenção sincera de entender os seus sentimentos e pensamentos demonstra um comportamento empático, assim como conhecer as próprias fraquezas e limites, permite o auto-reconhecimento.

Portanto, é possível perceber que pessoas com a inteligência social desenvolvida não são, necessariamente, pessoas com o QI alto, apesar de muitas características de personalidade serem similares, mas pessoas que aprenderam com a interação com os outros a ter comportamentos empáticos e flexíveis. Para o melhor desenvolvimento de competências é necessária a total integração das inteligências social, emocional e cognitiva (Emmerling e Boyatzis, 2012).

De acordo com a Teoria da Inteligência DWRI, muitas pessoas de alto QI não possuem a capacidade para desenvolver bons relacionamentos com os outros, sejam com pessoas com QI alto e sejam com pessoas com o QI mais baixo. Inteligência social não é consequiência exclusiva de um alto QI. Isso é resultado da incapacidade que elas possuem de controlar as suas emoções em situações de estresse, o que acaba culminando em conflitos relacionais, ansiedade e insegurança (Rodrigues, 2021).

Por esta razão, a inteligência social é fundamental para a formação de bons líderes. De acordo com Daniel Goleman (2008), as pessoas que possuem essa inteligência são capazes de se tornar líderes em seus ambientes de trabalho, familiar ou em alguma atividade em grupo, possuindo grande empatia e conhecimentos tanto próprio quanto dos estados mentais e emocionais dos outros. Estudos mostram que quando pessoas interagem com as outras, essa interação estimula a bioquímica do cérebro. Essa capacidade de liderança está menos relacionada com a quantidade de conhecimento ou o cargo de autoridade que a pessoa ocupa, mas sim na habilidade que esse líder tem de mostrar interesse genuíno para com as pessoas e fomentar nelas sentimentos positivos, mostrando que elas são necessárias e importantes. É a capacidade de inspirar outras pessoas.

Os melhores líderes têm a capacidade para reconhecer padrões de comportamento, padrões emocionais, de fazer críticas positivas sem prejudicar relacionamentos, antecipar as reações dos outros e antecipar e resolver situações de crise. Todas essas habilidades sociais podem ser aprendidas com as interações sociais, com a presença de um bom mentor e com a própria vontade de se conhecer e melhorar o próprio comportamento.

Seus estudos mostram que a inteligência social não é apenas uma questão da personalidade, mas uma questão das neurociências. O cérebro está envolvido nessa 
atividade.

Um dos fenômenos para que essa ligação entre líderes e seus seguidores aconteça é o fenômeno dos neurônios espelho. Os neurônios espelhos são responsáveis pelo processo de imitação, de mudanças faciais por ações quando estão olhando as ações de outros indivíduos e que essas ações sejam significativas para quem esteja olhando. Quando o cérebro de uma pessoa detecta as ações e emoções de outras, elas se ligam e “compartilham" a experiência (Ferreira et al, 2017).

Outro exemplo é o papel importante que o encorajamento exerce nas relações sociais. O encorajamento dos alunos dado por seus professores permite uma instrução mais efetiva em sala de aula, os alunos se sentem motivados e apoiados o que permite uma conexão maior entre eles e seus professores (Arghode, 2013).

A inteligência social é a base para a competência pedagógica e é vista como a capacidade fundamental para o sucesso do educador no seu trabalho. Educadores com essa inteligência possuem melhor competência comunicativa, adaptativa e nas suas relações interpessoais (Yermentaeyeva et al, 2014).

A inteligência social permite a prática da uma habilidade importante muitas vezes esquecida no nosso tempo: a humildade para aprender uns com os outros. Ser inteligente é saber que não sabe de tudo e que o outro pode ensinar. Aprendizagem é uma ação ativa, uma escolha consciente. Essa ação é benéfica para as relações humanas pois, desenvolve a confiança entre os membros de um sistema específico seja um grupo familiar, institucional ou praticante de uma atividade específica, uma comunidade, uma sociedade, onde todos têm a capacidade de mostrar o seu potencial e se sentirem valorizados (Reed et al, 2010).

Esse aprendizado pode ocorrer em um nível superficial ou envolve uma mudança conceptual e comportamental profundas. Atualmente, as interações sociais não estão mais delimitadas ao espaço físico. O advento da internet permitiu a formação de networks, redes de contato em que os participantes se ajudam, colaboram para alcançar algum objetivo em comum, aprendem uns com os outros, trocam de informações e compartilham seus conhecimentos (Becker et al, 2017).

Os resultados da aprendizagem são múltiplos. Quanto mais se aprende mais autoconfiante é a pessoas e menos ansiosa ela se torna. Ela não vê mais o outro como um concorrente e 
entende de maneira resolvida as próprias limitações aceitando que não sabe de tudo e que o outro pode ensinar.

A aprendizagem não é apenas capaz de modificar o indivíduo de maneira emocional e comportamental, mas também neurológica. A neuroplasticidade é a capacidade do cérebro de se reorganizar, mudar as suas funções e ligações em resposta a estímulos internos e externos, essa transformação ocorre desde células até comportamentos como consequência. Quanto mais se aprende mais o cérebro muda (Cramer et al, 2011).

\section{CONSIDERAÇÕES FINAIS}

A inteligência social é a forma como o indivíduo se comporta em uma sociedade e a sua interação com as demais pessoas. Para o seu desenvolvimento é importante o autoreconhecimento, ou seja, conhecer suas próprias capacidades, suas competências e seus limites. Buscar acreditar em si mesmo, com base na capacidade proveniente do conhecimento, sem medo de se posicionar, falar, interagir com os outros atingindo a plenitude sem se preocupar com o que os outros pensam, a não ser que o pensamento crítico interfira na razão para o aperfeiçoamento intelectual.

É natural do ser humano a disputa e a competição, porém, os indivíduos que desenvolvem a inteligência social se sentem confiantes e satisfeitos consigo mesmo, não entrando em disputas, que é proveniente do instinto pela sobrevivência herdada pela evolução. São capazes de confiar mais nas pessoas, praticam a humildade, aprendem com o outro e se aprimoram. Esse aprendizado permite a ativação dos neurotransmissores da recompensa e que promovem a plenitude, impedindo a ansiedade, a insegurança e emoções que promovam memórias negativas.

Quanto mais o indivíduo interage e aprende com os outros, mais as funções cognitivas se desenvolvem com a plasticidade neuronal. Assim, essa ampliação da inteligência permite que o indivíduo entenda melhor os desejos, as emoções e o limite do outro, percebe melhor as nuances a sua volta e se adapta tomando as melhores decisões com a ajuda do raciocínio lógico.

\section{REFERÊNCIAS}

Abas, N. A. H., Surdick, R., Otto, K., Wood, S., \& Budd, D. (2010). Emotional intelligence and conflict management styles (Doctoral dissertation, University of Wisconsin-Stout). 
Alhadabi, A., \& Karpinski, A. C. (2020). Grit, self-efficacy, achievement orientation goals, and academic performance in University students. International Journal of Adolescence $\quad$ and $\quad$ Youth, 25(1), 519-535. https://doi.org/10.1080/02673843.2019.1679202

Arghode, V. (2013). Emotional and social intelligence competence: Implications for instruction. International Journal of Pedagogies and Learning, 8(2), 66-77.

Badea, L., \& Pană, N. A. (2010). The Role of Empathy in Developing the Leader's Emotional Intelligence. Theoretical \& Applied Economics, 17(2).

Becker, J., Brackbill, D., \& Centola, D. (2017). Network dynamics of social influence in the wisdom of crowds. Proceedings of the national academy of sciences, 114(26), E5070-E5076.

Boyatzis, R. E., Thiel, K., Rochford, K., \& Black, A. (2017). Emotional and social intelligence competencies of incident team commanders fighting wildfires. The Journal of Applied Behavioral Science, 53(4), 498-516.

Chopra, P. K., \& Kanji, G. K. (2010). Emotional intelligence: A catalyst for inspirational leadership and management excellence. Total quality management, 21(10), 9711004. https://doi.org/10.1080/14783363.2010.487704

Cramer, S. C., Sur, M., Dobkin, B. H., O'brien, C., Sanger, T. D., Trojanowski, J. Q., ... \& Vinogradov, S. (2011). Harnessing neuroplasticity for clinical applications. Brain, 134(6), 1591-1609. https://doi.org/10.1093/brain/awr039

de Abreu Rodrigues, F. (2021). Pessoas muito inteligentes são uma ameaça. Ciencia Latina Revista Científica Multidisciplinar, 5(5), 9850-9857.

de Abreu Rodrigues, F. (2021). DWRI Intelligence and Other Intelligences. International Journal of Development Research Vol. 11, Issue, 01, pp. 43576-43584, January. https://doi.org/10.37118/ijdr.20911.01.2021

Emmerling, R. J., \& Boyatzis, R. E. (2012). Emotional and social intelligence competencies: cross cultural implications. Cross Cultural Management: An International Journal.

Ferreira, V. R. T., Cecconello, W. W., \& Machado, M. R. (2017). Neurônios-espelho como possível base neurológica das habilidades sociais. Psicologia em Revista, 23(1), 147-159. 
Gillebaart, M. (2018). The 'operational'definition of self-control. Frontiers in psychology, 9, 1231. 18 July 2018 | https://doi.org/10.3389/fpsyg.2018.01231

Goleman D. \& Boyatzis, R. E. (2008). Social Intelligence and the Biology of Leadership. Harvard Business Review. https://hbr.org/2008/09/social-intelligence-and-thebiology-of-leadership

Hatala, K., Roach, N., Ostrofsky, K. et al. Footprints reveal direct evidence of group behavior and locomotion in Homo erectus. Sci Rep 6, 28766 (2016). https://doi.org/10.1038/srep28766

Le Deist, F. D., \& Winterton, J. (2005). What is competence?. Human resource development international, 8(1), 27-46.

Lievens, F., \& Chan, D. (2010). Practical intelligence, emotional intelligence, and social intelligence.

Kihlstrom, J. F., \& Cantor, N. (2000). Social intelligence. In R. J. Sternberg (Ed.), Handbook of intelligence (pp. 359-379). Cambridge University Press. https://doi.org/10.1017/CBO9780511807947.017

Reed, M. S., Evely, A. C., Cundill, G., Fazey, I., Glass, J., Laing, A., ... \& Stringer, L. C. (2010). What is social learning?. Ecology and society, 15(4).

Rightmire, G. Philip (2020, November 12). Homo habilis. Encyclopedia

Stricker, L. J., \& Rock, D. A. (1990). Interpersonal competence, social intelligence, and general ability. Personality and Individual Differences, 11(8), 833-839.

Tadayon, E., Pascual-Leone, A., \& Santarnecchi, E. (2020). Differential contribution of cortical thickness, surface area, and gyrification to fluid and crystallized intelligence. Cerebral Cortex, 30(1), 215-225.

Webb, C. A., Weber, M., Mundy, E. A., \& Killgore, W. D. (2014). Reduced gray matter volume in the anterior cingulate, orbitofrontal cortex and thalamus as a function of mild depressive symptoms: a voxel-based morphometric analysis. Psychological medicine, 44(13), 2833-2843.

Westli, H. K., Johnsen, B. H., Eid, J., Rasten, I., \& Brattebø, G. (2010). Teamwork skills, shared mental models, and performance in simulated trauma teams: an independent group design. Scandinavian journal of trauma, resuscitation and emergency medicine, 18(1), 1-8. 
Yermentaeyeva, A., Aurenova, M. D., Uaidullakyzy, E., Ayapbergenova, A., \& Muldabekova, K. (2014). Social intelligence as a condition for the development of communicative competence of the future teachers. Procedia-Social and Behavioral Sciences, 116, 4758-4763. 